\title{
Évolution ou changements radicaux ?
}

\author{
Bernard Vermot-D esroches \\ Université du Q uébec à Trois-Rivières
}

\section{Introduction}

Le développement des régions au Québec, une grande question qui de prime abord peut sembler trop évidente, voire ennuyeuse. C'est pourtant un point de base que l'on ne devrait jamais tenir pour acquis. Effectivement, c'est ce type de question que sans arrêt nous devrions mettre en avant pour être certains de ne jamais s'égarer dans des sous-objectifs plus embrouillés.

\section{Un peu d'histoire pancanadienne}

Observée de tous côtés, la «machine québécoise » révèle à coup sûr ses dépendances ou ses liens avec son environnement canadien et américain. On ne peut ignorer aussi facilement d'aussi grands voisins.

Le Canada constitue l'un des espaces géographiques où le phénomène des régions et du régionalisme est le plus développé. Les causes premières sont évidentes et se rapportent dans un premier temps à la physiographie du pays ; Cole Harris compare le Canada à un «océan d'ombre parsemé d'îles de lumière », constituant un archipel qui s'étire sur 6500 kilomètres d'est en ouest. Ce sont aussi et surtout les gens, les nations indigènes et les groupes multiethniques immigrant depuis bientôt quatre siècles qui constituent le relief des paysages régionaux canadiens. A. Reynaud considère dans une perspective internationale ce pays comme l'un des plus diversifiés sur le plan de la géopolitique.

Depuis la naissance de la Confédération, et même bien avant, la reconnaissance des régions et de leurs particularismes s'est toujours imposée comme le cheval de bataille par excellence des hommes politiques canadiens, tant sur le plan fédéral que provincial.
Récemment, le Premier Ministre du Canada affirmait que la lutte pour restreindre l'ampleur des disparités régionales devait demeurer l'objectif premier et fondamental de toute politique économique canadienne.

Le Québec se retrouve dans ce grand enchevêtrement régional dont l'importance se trouve amplifiée par ses caractéristiques personnelles et distinctes du reste du continent. Au-delà de la composante politique que l'on peut prêter facilement à tout homme public tenant ce langage persiste néanmoins une vérité profondément enracinée dans l'âme des Québécois : par son histoire, sa géographie, ses cultures et son économie, le Québec s'impose aussi comme une mosaïque disparate composée d'entités inégales et vouées à des développements inégaux. La région de Salluit dans le GrandNord de notre province n'a aucun point de comparaison sensé avec les régions « sudistes ». Mais préoccupons-nous davantage de ces régions qui s'étendent à moins de 300 kilomètres de la frontière américaine et qui renferment plus de $95 \%$ de la population.

Idéalement, le développement régional devrait s'identifier au développement national. On collabore avec les proches pour développer un espace qui n'a comme limites que les frontières administratives. Mais les Québécois, comme bien d'autres, ne travaillent que pour l'espace leur appartenant immédiatement. On aime beaucoup quelques cousins éloignés mais on s'occupera d'abord de ses frères proches. Le résultat immédiat est plus stimulant. 
Idéalement, le développement régional devrait s'identifier au développement national. On collabore avec les proches pour développer un espace qui n'a comme limites que les frontières administratives. Mais les Québécois, comme bien d'autres, ne travaillent que pour l'espace leur appartenant immédiatement. On aime beaucoup quelques cousins éloignés mais on s'occupera d'abord de ses frères proches. Le résultat immédiat est plus stimulant. L'ouvrage de Luc Bureau, Entre l'eden et l'utopie, décrit avec humour quelques exemples purement québécois.

\section{Les particularités québécoises}

Développer une région au Québec revient au même exercice que celui qui se pratique dans le cadre de tout autre développement régional classique. Les deux grandes variables économiques qui définissent le développement régional sont l'emploi et la richesse. Ces deux variables ne sont d'ailleurs pas seulement de nature économique ; un individu privé de son emploi, donc appauvri, risque fort d'avoir d'autres problèmes, psychologiques, sociaux, etc. Sans emploi et sans richesse, une région stagne, se replie, se démobilise et parfois meurt. Les exemples de Schefferville et de Gagnon sont éloquents à cet égard. Le développement d'une région ne se définit pas que par l'économie, nous venons de le mentionner. Le social occupe également une dimension très respectable.

Dans ce cadre habituel, le Québec joue avec son dynamisme propre, ses particularités géographiques et sociales. Le pays est immense, même dans sa zone sud. Près de 2000 kilomètres séparent la BasseCôte-Nord de Windsor sur une petite largeur de 300 kilomètres. Une sorte de «Chili horizontal» qui provoque des ignorances interrégionales et donc, des différences.

Cette disposition géographique a des origines historiques indéniables, mais configure la province en zones socialement quasi indépendantes et économiquement concurrentes. L'Estrie est issue des loyalistes britanniques qui fuyaient la nouvelle Amérique ; l'Abitibi est issue d'une véritable volonté de développement et d'expansion. Mais à l'heure actuelle, quels points communs pourrait-on faire ressortir entre le Témiscamingue et la Gaspésie, ou même entre Trois-Rivières et Trois-Pistoles ou encore la Montérégie ? Dans ce sens, en Europe, les liens entre Lyon et Marseille ou Mayence et Hambourg sont beaucoup plus sensibles.

La préoccupation régionale est forte par nature. Bien sûr, l'appartenance même au lieu où se réalisent toutes les activités est mobilisatrice et créatrice. Le développement global de la « Nation», de la province, est plus diffus, souvent inexistant. En revanche, la concurrence interrégionale, parfois fortement développée à l'intérieur de zones bien spécifiques (par exemple le Saguenay - Lac-Saint-Jean) peut être positive dans le sens où elle mobilise et favorise des activités novatrices et enrichissantes. Un duel peut être négatif s'il s'isole et se détourne des préoccupations supérieures, mais il peut enrichir en exacerbant les volontés. Le Centre-du-Québec, cette nouvelle région administrative, complète et parfois concurrence à ravir la région de l'Estrie. Les «appartenances » à l'une ou à l'autre sont tellement proches.

Le bilan est clair, le Québec se développe globalement parce qu'il est animé de particules dynamiques. L'addition de ces particules est certes positive mais donne des résultats inférieurs à ceux que fournirait cette « Sur-additivité » générée par l'interdépendance naturelle. Notre gouvernement en est tout à fait conscient et cherche depuis longtemps à contrer ces vagues d'isolationnisme.

Le bilan est clair, le Québec se développe globalement parce qu'il est animé de particules dynamiques. L'addition de ces particules est certes positive mais donne des résultats inférieurs à ceux que fournirait cette «sur-additivité » générée par l'interdépendance naturelle. Notre gouvernement en est tout à fait conscient et cherche depuis longtemps à contrer ces vagues d'isolationnisme. L'ancien OPDQ (Office de planification et de développement du Québec) le démontre. Déjà dans les années 1960, les ministères intégraient des services régionaux. Depuis une dizaine d'années, les régions font partie intégrante d'un organisme officiellement intégrateur : le ministère des Régions. Cet organisme est tout à fait vital pour les micro-régions. Elles y sont reconnues et considérées au même titre que les grandes. Ces grandes régions en tirent également des avantages d'effet de taille. Mais la dimension 
intégration est trop souvent négligée parce que mal perçue.

Actuellement, le problème fondamental se formule à ce niveau de perception ainsi qu'à celui du niveau optimal de bien-être. Et surtout, les nouvelles technologies de l'information, des communications et des échanges perturbent ces perceptions.

Pourquoi se donner la peine d'échanger si le niveau de développement est déjà accepté et même écarté de toute problématique ? Il y a quelques années, les mécontents le faisaient savoir et favorisaient les prises de conscience. Une région n'était pas toujours informée, et dans l'ignorance, les besoins sont moins évidents; ils ne ressortent qu'en cas de crise et de difficultés immédiates ou permanentes. Maintenant, ces mécontents déménagent, et si l'appartenance les incite parfois à demeurer dans leur région, ils peuvent quand même «virtuellement » quitter la région et satisfaire les besoins de communication et d'échange qu'ils éprouvent. Le régionalisme est à la fois victime et profiteur de ce nouveau phénomène «NTIC ». Les régions sont à la fois techniquement rapprochées et humainement éloignées. Les habitants voyagent encore, mais pour le loisir et le tourisme. Le social les tient encore, mais l'économique, bien qu'il ne cesse de grandir, se diffuse en se dispersant dans l'espace géographique et électronique.

Le régionalisme est à la fois victime et profiteur de ce nouveau phénomène «NTIC». Les régions sont à la fois techniquement rapprochées et humainement éloignées. Les habitants voyagent encore, mais pour le loisir et le tourisme. Le social les tient encore, mais l'économique, bien qu'il ne cesse de grandir, se diffuse en se dispersant dans l'espace géographique et électronique.

\section{D es évolutions ou des changements radicaux}

La balle est lancée, le lourd passé régional du Québec a bâti une province très particulière, mais le présent bouleverse à toute allure ces acquis déjà jugés immuables.
En effet, la dynamique a radicalement changé. On assiste simultanément à de nouvelles interactions qui évoquent une sorte de dérégionalisation, et à un resserrement de plus en plus marqué de la politique régionale au Québec.

\section{La pulvérisation des anciennes interactions}

Phénomène évident allant depuis plusieurs années en perpétuelle augmentation. Il n'est plus nécessaire d'habiter Montréal pour travailler à Montréal. Il n'est plus nécessaire de travailler à Trois-Rivières pour étudier à Trois-Rivières. Les anciennes interactions ne pouvaient se réaliser que dans un cadre étroit, planifié et rentabilisé au maximum; les erreurs pouvaient coûter cher. Ces interactions se faisaient sur de courtes distances et dans des espaces réduits. Ces échanges économiques étaient évidents en raison de la fréquence des véhicules utilisés, du poids de la marchandise transportée, des contraintes des saisons et surtout, du respect des appartenances ancrées dans l'esprit des gens.

Depuis quelque temps, ces interactions se diffusent dans des régions de plus en plus éloignées. Chaque territoire échange avec le reste de la province et souvent avec le monde, donc à l'échelle internationale. Une région n'interagit plus qu'avec quelques autres régions privilégiées et tout à fait prévisibles. Le commerce international a littéralement explosé, favorisant du coup des échanges spatialement plus diversifiés. Les raisons sont connues : les camions sont plus gros et plus efficaces, les routes sont en meilleure condition, les séquelles du climat sont amoindries et le sentiment d'appartenance s'est radicalement transformé. Ces facteurs conduisent simultanément les activités d'interaction vers une plus grande efficacité d'ensemble. Ceci a mené tout naturellement au rétrécissement « virtuel» de l'espace économique auquel nous assistons maintenant.

\section{L'exemple évident du système universitaire québécois}

Une dizaine d'institutions forment le réseau principal des universités québécoises. Chacune d'elles demeure reliée géographiquement à sa région, mais elles se disputent des sous-espaces de plus en plus communs, la clientèle étudiante et d'autres activités d'intervention (recherche, implications dans le milieu, etc.). 
À titre d'exemple, près de la moitié des étudiants de l'Université du Québec à Trois-Rivières provient des autres régions de la province et des autres régions $\mathrm{du}$ monde. Naturellement, la diversification des produits et les spécialités de chacune justifient ces répartitions, mais celles-ci n'auraient pu se réaliser aussi facilement il y a seulement vingt-cinq ans. À partir de ce petit exemple simple et connu, on peut comprendre rapidement toute l'importance de ce phénomène.

Une dizaine d'institutions forment le réseau principal des universités québécoises. Chacune d'elles demeure reliée géographiquement à sa région, mais elles se disputent des sous-espaces de plus en plus communs, la clientèle étudiante et d'autres activités d'intervention (recherche, implications dans le milieu, etc.).

\section{Un nouveau découpage}

Le Québec est souvent pris comme exemple de tel ou tel type de découpage régional (régions-programmes, MRC, municipalités, etc.). On vit dans un espace assez grand, ni trop isolé ni trop dépendant. Les contraintes politiques ou légales sont quasi inexistantes. De plus, le peuple est habitué depuis longtemps, historiquement, à s'adapter à des changements importants et rapides.

La fameuse idée «centre - périphérie » renaît de ses cendres depuis quelques années. Elle renaît non plus en mettant de l'avant un espace urbain et industriel parfaitement délimité qui va dominer une périphérie rurale, mais en mettant en évidence les hiérarchies de dominances économiques indépendamment des espaces impliqués.

L'espace n'est donc pas entièrement disparu ; son rôle a diminué mais on assiste surtout à des transformations très significatives. Les grandes villes sont devenues interdépendantes de leurs couronnes immédiates, et les villes moyennes, de leurs larges couronnes plus périphériques.

\section{Une contradiction apparente}

Le développement régional au Québec transite depuis quelque temps d'une rive à l'autre du grand courant des opinions politiques conduisant aux réformes structurelles.

\section{Une «politique régionale» pourtant claire}

La philosophie régionaliste n'est pas neuve ni au Québec, ni ailleurs dans le monde. Sans remonter trop loin dans le temps, on se souvient encore des exercices, comme ceux du Comité de mesure d'urgence pour la Gaspésie pratiqués dans les années 1950, ou d'expériences plus malheureuses comme celle du Bureau d'aménagement de l'est du Québec (BAEQ). À partir du milieu des années 1980, cette volonté de considérer les différences régionales se matérialise concrètement par le rattachement des «affaires » régionales au ministère de Marc-Yvan Côté. En 1992, on assiste à la disparition du vieil OPDQ au profit d'une régionalisation «interministérielle » plus évidente. Depuis quelques années, la province possède son véritable ministère des Régions, dont les préoccupations régionales sont beaucoup plus évidentes que celles de l'OPDQ, qui se contentait de jouer avec les disparités.

Grâce à cette réforme, les CRD (conseils régionaux de développement) et les CLD (centres locaux de développement) déjà en place ont pu bénéficier d'une relance évidente. La politique rurale est également mise de l'avant avec plus d'évidence. Il convient surtout de remarquer la naissance et le développement naturel de pouvoirs locaux utiles et respectés. Les comités de quartiers urbains, les CLD ou les organismes plus temporaires mais encore plus ciblés sont nombreux et présents. Une émergence semble très claire : celle de la volonté des reconnaissances des identités et des appartenances. Une contradiction bien humaine : celle du besoin de différenciation et du besoin de nivellement. L'évolution économique et technologique force des rapprochements et des mariages qui sont de nature à niveler les particularités personnelles et régionales. Ce besoin vital de différenciation est donc exacerbé.

On assiste donc à un renforcement d'une sorte de gouvernement régional et local qui se concrétise de plus en plus. Le gouvernement fédéral joue également dans cet esprit. Les SADC (sociétés d'aide au développe- 
ment des collectivités) et d'autres regroupements plus sectoriels vont dans ce sens.

Les comités de quartiers urbains, les CLD ou les organismes plus temporaires mais encore plus ciblés sont nombreux et présents. Une émergence semble très claire : celle de la volonté des reconnaissances des identités et des appartenances. Une contradiction bien humaine : celle du besoin de différenciation et du besoin de nivellement. L'évolution économique et technologique force des rapprochements et des mariages qui sont de nature à niveler les particularités personnelles et régionales.

\section{Mais une intégration marquée}

Conscient et spectateur de ces rapprochements évidents dictés par l'efficacité que génère le progrès technologique, notre gouvernement retrouve son rôle de leader en menant ou en obligeant des mouvements importants d'intégration.

Le plus évident a été lancé en 1999 dans cette grande opération de «démunicipalisation». Les raisons sont claires mais les oppositions que ce mouvement a fait apparaître sont tout à fait claires également. L'économie et l'efficacité en général sont primordiales bien sûr, à condition que l'identité et les liens fondamentaux d'appartenance soient respectés. La frontière est cependant trop étroite, et des chevauchements inévitables font apparaître parfois de fortes dissensions qui se répercutent jusqu'au niveau des élections. Le slogan «Une île une ville » est remarquable à ce chapitre. Les dernières élections municipales ont été clairement orientées par rapport à cette problématique. L'arrêt subit de cette «démunicipalisation» est probablement un cas typique d'une politique d'accompagnement.

Les regroupements municipaux ne sont pas un mouvement isolé. Les conglomérats d'entreprises se forment rapidement et sont de plus en plus fréquents. Les exemples des télécommunications ou des transports sont révélateurs. On assiste effectivement à un retour vers un centralisme économique. Les premiers com- munistes auraient parlé de monopolisation grandissante qui engendrera bientôt la fin apocalyptique du capitalisme prévue par Karl Marx.

Un problème de taille pour les philosophes, les économistes et les régiologistes. Une solution qui devra contourner cette grande contradiction en allant peut-être vers une définition plus dynamique de la région. La région «perçue » qu'Antoine Bailly tirait de sa Géographie de la perception répondrait peut-être mieux à cette vaste problématique. Bref, gardons nos limites administratives qui prennent de l'importance au fil du temps (les départements français et les Landkreis allemands en sont des exemples), elles sauront satisfaire nos besoins humains d'appartenance, et n'hésitons pas à élargir la région économique aussi loin que les nouvelles technologies et l'efficacité économique sauront nous conduire. Il ne s'agira peutêtre plus de mariage, mais bien de concubinage désiré.

\section{Et notre véritable développement régional actuel}

Dans le cadre multidimensionnel que nous venons d'évoquer, le développement de nos régions n'est ni moribond, ni malade. Peut-être un peu essoufflé et parfois résigné. Nos MRC, relativement jeunes avec leurs vingt ans d'existence, sont probablement les entités administratives actuellement les plus réalistes, tant sur le plan du critère de l'appartenance que sur celui de l'efficacité économique.

Les MRC urbaines se portent bien et évoluent dans le même sens que leurs homologues américaines ou européennes. Les MRC rurales s'adaptent relativement bien à chacune de leurs situations particulières. Si elles sont limitrophes des autres provinces, comme celle du Témiscamingue ou celle d'Avignon en Gaspésie, elles tirent alors profit des autres centres canadiens comme New Liskeard en Abitibi ontarienne ou Campbellton dans le nord du NouveauBrunswick. Si elles sont intermédiaires comme celles de la nouvelle région 17, le Centre-du-Québec, elles vont exploiter leur situation de nœud (Trois-Rivières Sherbrooke - Québec - Montréal). Si elles sont excentrées comme celles de Caniapiskau ou de la Vallée de l'Or, elles exploiteront alors au mieux leurs différences, qui les rendent uniques et complémentaires. Bien sûr, quelques-unes devront se résigner à simplement survivre. Le Grand-Nord québécois ou la muni- 
cipalité de la Basse-Côte-Nord, collée au Labrador, ne peuvent pas s'attendre à un miracle économique dans l'immédiat.

Le développement de nos régions n'est ni moribond, ni malade. Peutêtre un peu essoufflé et parfois résigné. Nos MRC, relativement jeunes avec leurs vingt ans d'existence, sont probablement les entités administratives actuellement les plus réalistes, tant sur le plan du critère de l'appartenance que sur celui de l'efficacité économique.

Des études que nous avons récemment menées démontrent qu'il n'y a pas augmentation du développement régional, travail et richesse, dû à une accélération des échanges interrégionaux immédiats. Les échanges entre les MRC ont effectivement globalement augmenté depuis 1992, mais selon des répartitions géographiques peu prévisibles. Les désenclavements successifs de l'ALENA de la CEE, ceux prodigués par l'Organisation mondiale de commerce sont venus favoriser les effets naturels qu'engendraient les nouvelles technologies et les nouvelles mentalités.

Le vieux débat déconcentration décentralisation demeure cependant la pierre d'achoppement de bien des régions. Celles-ci acceptent cette idée attirante de régionalisation, de décentralisation, voire d'autonomie, mais réalisent vite que la quantité de compétence réclamée est assez impressionnante. Un glissement imperceptible mais continu vers la déconcentration s'opère alors automatiquement.

Le vieux débat déconcentration - décentralisation demeure cependant la pierre d'achoppement de bien des régions. Celles-ci acceptent cette idée attirante de régionalisation, de décentralisation, voire d'autonomie, mais réalisent vite que la quantité de compétence réclamée est assez impressionnante. Un glissement imperceptible mais continu vers la déconcentra- tion s'opère alors automatiquement. Le Québec n'a pas de régions véritablement orphelines. Malheureusement, quelques-unes d'entre elles pourraient mourir économiquement comme la ville de Gagnon est morte dans les années 1970. Fernand Martin semble avoir raison sur ce point : toutes les régions ne sont pas viables.

\section{En conclusion: le pouvoir et le territoire}

Les surconnexions et les mélanges obligés qu'entraînent les nouvelles technologies sont incontournables si l'on veut s'inscrire dans un processus de développement régional classique. Ce phénomène est d'autant plus inévitable qu'il est intimement lié au pouvoir. Un pouvoir qui est à l'origine même de l'idée de région. Même une région perçue évoque l'idée de pouvoir. Un regroupement met en valeur, informe et autorise à un contrôle, donc à un pouvoir éventuel.

Un regroupement, une localité, une région ancienne possède une forme de pouvoir que le temps a fait acquérir naturellement. Ces regroupements obligés auxquels nous assistons ces derniers temps permettent de maintenir le pouvoir acquis antérieurement. Ceci n'est jamais évoqué directement ; on parlera davantage d'éléments que nous avons déjà mentionnés tels l'appartenance ou l'efficacité. Cependant, cette notion de pouvoir régional ou local est toujours sousjacente.

Le besoin de remodeler le paysage régional est là, les régions se pulvérisent, se virtualisent économiquement. Socialement, les notions habituelles de géographie comme celle d'appartenance restent en place mais éprouvent, par voie de conséquence, ce besoin de remodelage.

De ce grand tourbillon où se trouvent confrontés les acquis, la poussée des NTIC, les besoins de s'adapter, émerge un besoin fondamental, non discutable, originel à tous égards : celui de se différencier.

À notre avis, cette différenciation se fonde sur :

- les opportunités, les chances qu'un territoire peut obtenir au hasard des interrelations économiques. Par exemple, le lien que l'on fait depuis quelques années entre Montréal et le 
développement des NTIC est très représentatif. Dans ce cas, le hasard s'appelle peut-être Softimage ou autre chose, mais il n'était pas prévisible ;

- le comportement psycho-organisationnel qu'une personne un tant soit peu en affaires va développer instinctivement. La différenciation est naturelle, elle permet la relativisation des choses et la mise en valeur de la personne impliquée.

Les événements qui se déroulent actuellement à l'échelle des municipalités, ces nouvelles idées de « territorialisation » sont donc la manifestation d'une opposition entre l'uniformisation tendancielle et le besoin naturel de différenciation.

De nombreux exemples confirment cette réflexion. Le cas de Montréal a déjà été mentionné plus haut. Bien sûr, il s'agit d'une très grosse région urbaine qui concerne globalement près de la moitié du Québec. Cependant, les sous-régions et les différentes couronnes de l'agglomération vont dans le même sens. L'autre grande région urbaine, celle de Québec, subit un phénomène analogue.

Si l'on sort un peu de la province, on retrouve d'autres exemples urbains comme le cas de Windsor - Détroit, et si l'on se tourne vers les territoires ruraux plus éloignés des grands centres, ce mouvement de développement généré par ce moteur qui fonctionne grâce à l'opposition que l'on vient d'évoquer est également présent. La région du Saguenay - LacSaint-Jean en est tout à fait représentative.

En 2001, le régionalisme est toujours présent, important, créateur mais considérablement transformé et mdapté aux réalités technologiques et 